\title{
Aerobic exercise is an independent determinant of levels of inflammation and oxidative stress in middle-aged obese females
}

\author{
Kyung-Shin Park*, Brett S. Nickerson \\ Kinesiology, Texas A\&M International University, Laredo, TX, USA
}

The purpose of this study was to investigate the effects of a 4-week moderate-intensity aerobic exercise on changes of body composition and markers of inflammation and oxidative stress independent from weight loss in middle-aged obese females. Thity-five obese females were randomly assigned to either an exercise $(E X, N=16)$ or control (CON, $\mathrm{N}=19$ ) group. The EX performed moderate intensity aerobic exercise on the treadmill for $60 \mathrm{~min}$ at $55 \%$ of maximal oxygen consumption $\left(\mathrm{VO}_{2 \text { max }}\right)$ for 4 weeks ( 3 days/wk). Body composition measurement with dual-energy $\mathrm{x}$-ray absorptiometry and blood collection were conducted before and after the 4-weeks intervention. Blood samples were used to measure levels of tumor necrosis factor-alpha (TNF- $\alpha$ ), C-reactive protein, adiponectin, total antioxidant status (TAS), and 8-hy- droxydeoxyguanosine. Four weeks of aerobic exercise intervention significantly increased $\mathrm{VO}_{2 \max }$ in $\mathrm{EX}(P<0.001)$. EX also observed a decrease in TNF- $\alpha(P=0.033)$ and an increase in TAS $(P=0.028)$ without changes in body weight and fat mass after 4 weeks of aerobic exercise training. No changes were observed in CON after the intervention. Results of this study indicate that moderate aerobic exercises may contribute, at least a part, to reductions of inflammation and oxidative stress independently from fat loss. Therefore, it may reduce risks of obesity-associated disorders in middle-aged obese females.

Keywords: Aerobic exercise, Obesity, Body composition, Inflammatory cytokines, Oxidative stress

\section{INTRODUCTION}

The prevalence of obesity and obesity-related diseases has increased significantly over the past two decades to pandemic proportions (Cao, 2014; Tumova et al., 2013). Obesity is considered to increase the risk for many diseases such as cancers, cardiovascular disease, and type 2 diabetes mellitus (Bell et al., 2014; Hatoum et al., 2012), which often results from lack of exercise, good diet, and maintaining a healthy lifestyle (Pérez-Escamilla et al., 2017; van Rossum, 2017).

Excess body fat, acting as an active endocrine organ, is associated with elevated levels of proinflammatory cytokines and oxidative stress in the blood (Beavers et al., 2015; Bruun et al., 2006) which are known to induce cancers, type 2 diabetes, and cardiovascular diseases. Therefore, the inflammatory pathway with oxi- dative stress is pointed out by researchers as a potential therapeutic approach targeting for lifestyle interventions designed for obese populations to reduce diseases and disabilities (Wang and He, 2018; You et al., 2004; Zatterale et al., 2020).

There is mounting evidence that aerobic exercise is as an effective and preventive countermeasure to chronic diseases. Previous studies reported that aerobic exercise training reduced cardiovascular risk factors and improved insulin sensitivity, which was accompanied by reductions in proinflammatory cytokines and oxidative stress in overweight and obese population (Abramson and Vaccarino, 2002; Adamopoulos et al., 2001; Church et al., 2002; Conraads et al., 2002). Results of these studies suggest that aerobic exercise training may improve the inflammatory profile and oxidative stress independently from weight loss. However, most studies failed to confirm the effects of exercise on inflammatory
*Corresponding author: Kyung-Shin Park (DiD https://orcid.org/0000-0002-0761-4502 Kinesiology, Texas A\&M International University, 5201 University Blvd., Laredo, TX, USA

Email: kpark@tamiu.edu

Received: December 13, 2021 / Accepted: January 11, 2022
This is an Open Access article distributed under the terms of the Creative Commons Attribution Non-Commercial License (https://creativecommons.org/licenses/by-nc/4.0/) which permits unrestricted non-commercial use, distribution, and reproduction in any medium, provided the original work is properly cited. 
cytokines and oxidative stress (Beavers et al., 2015; Fatouros et al., 2004; Kelishadi et al., 2008; Marcell et al., 2005) independent from fat mass, therefore, it is generally accepted that a reduction in fat mass is required to induce decreases in markers of proinflammatory cytokines and oxidative stress.

Even though the mechanism which links exercise training to these inflammatory markers are not fully understood yet, a few recent studies provided evidence that physical activities may, independently from weight loss or fat mass, suppress oxidative stress (Samjoo et al., 2013), the production of inflammatory markers (Starkie et al., 2003), enhance the anti-inflammatory indices (You et al., 2013).

With given information of the relation between obesity and cardiovascular risk factors, insulin resistance, and inflammation, it is important to understand whether systemic inflammation and oxidative stress can be in part explained by the physiologic benefits of exercise and whether exercise can be used as a potential therapeutic intervention for overweight and obese individuals to prevent the prevalence of chronic diseases. Therefore, the purpose of this study was to investigate the effects of exercise training on inflammatory biomarkers in the absence of weight loss and fat loss by conducting a short-term, a 4-week exercise training intervention.

\section{MATERIALS AND METHODS}

\section{Subjects}

A total of 38 obese Hispanic females aged $34-46$ years old, were recruited from the Hispanic community in the United States. Participants were considered eligible if they were nonsmokers, premenopausal, obese (\% body fat $>30 \%$ ), physically inactive; did not have any known chronic disease; did not take medication that would alter metabolic, cardiovascular, or immune function; and had no musculoskeletal limitations. Participants who did not have menses during the last 2 months were excluded from this study. This study was approved by the Institutional Review Board (TAMIU-IRB-20131203) and the subjects provided written informed consent and the medical history forms prior to performing any study protocols.

\section{Study design}

During the initial visit, the subjects were randomly assigned to either an exercise (EX: $\mathrm{N}=19)$ or control $(\mathrm{CON}: \mathrm{N}=19)$ group. Subjects were assessed 2 times: before the intervention (PRE) and after the 4 weeeks of intervention (POST). Subjects were asked to maintain a normal dietary regimen and refrain from any forms of aerobic or anaerobic physical activity other than the exercise treatment throughout the study period. For each test, subjects arrived at the testing center between 08:00 and 09:00 hr after $12 \mathrm{hr}$ of fasting and underwent anthropometric measurements, measurement of body fat and visceral adipose tissue mass, blood sample collection, and maximal oxygen consumption $\left(\mathrm{VO}_{2 \max }\right)$ test. Total three subjects in exercise group were excluded due to missing more than 3 days of training. Therefore, a total of 35 subjects (16 EX, and $19 \mathrm{CON}$ ) completed this study.

\section{Anthropometric measurements}

All measurements were completed in duplicate by a trained technician. Height and weight were measured to the nearest 0.1 $\mathrm{cm}$ and $0.1 \mathrm{~kg}$, respectively, with the participants wearing indoor clothes without shoes.

\section{Body composition assessment}

Total and multicompartment body composition was measured by dual-energy x-ray absorptiometry (Hologic Discovery Series, Bedford, MA, USA) at PRE and POST. This allows for estimation of total lean mass and fat mass within specific regions, including the visceral depot. Subjects lied on the table and the entire body were scanned. The subject lied flat on her back for the duration of the scan without difficulty, pain, or shortness of breath. Subjects were asked to wear a gown and to remove all metal objects (glasses, jewelry, and cell phones). The scans were performed following the standard clinical protocol.

$\mathrm{VO}_{2 \max }$ test

$\mathrm{VO}_{2 \max }$ test was conducted in each testing day and the result of $\mathrm{VO}_{2 \max }$ test at PRE was used to determine exercise intensity $(55 \%$ of $\mathrm{VO}_{2 \max }$ ) for subjects in exercise group. $\mathrm{VO}_{2 \max }$ was measured during a continuous, progressive, treadmill running protocol on a level grade, with which subjects started to walk at $4 \mathrm{~km} / \mathrm{hr}$ and speed was increased $0.8 \mathrm{~km} / \mathrm{hr}$ every $2 \mathrm{~min}$.

\section{Blood collection and analyses}

Five milliliter of venous blood was drawn from an antecubital vein right after body fat measurement in each testing day. Collected blood sample was centrifuged in serum separating vacutainer tubes at $1,000 \times \mathrm{g}$ for $15 \mathrm{~min}$ (Allegra X-15R Refrigerated Centrifuge, Beckman Coulter, Irving, TX, USA). The serum was then stored at $-80^{\circ} \mathrm{C}$ until blood chemical analyses were conducted.

Tumor necrosis factor-alpha (TNF- $\alpha$ ), C-reactive protein (CRP), 
adiponectin, total antioxidant status (TAS), and 8-hydroxydeoxyguanosine $(8-\mathrm{OHdG})$ in blood were measured using the enzymelinked immunosorbent assay. All these variables were determined with commercial kits (Cayman Chemical Co., Ann Arbor, MI, USA) using a microplate reader (EL 808, BioTek Co., Winooski, VT, USA). The mean intra-assay coefficients of variation (CVs) were $6.5 \%, 5.8 \%, 5.6 \%$, and $6.7 \%$ and Interassay CVs were $6.9 \%$, $7.9 \%, 8.5 \%, 7.4 \%$, and $7.5 \%$ for TNF- $\alpha$, CRP, adiponectin, TAS, and $8-\mathrm{OHdG}$, respectively.

\section{Exercise intervention}

Subjects assigned to the exercise group performed walking exercise on a treadmill at a predetermined exercise intensity (heart rate at $55 \% \mathrm{VO}_{2 \max }$ ) for $60 \mathrm{~min}, 3$ days a week for 4 weeks. During each exercise session, the subjects' heart rate was monitored using the Polar heart rate monitor (Polar, Lake Success, NY, USA) and recorded every 10 min to ensure their target exercise intensity. As each subject's fitness level improved, the exercise speed on treadmill was increased on the basis of heart rate in order to maintain a heart rate at $55 \% \mathrm{VO}_{2 \max }$. Speed was adjusted when average heart rate was decreased by more than 5 beats per minute on 2 consecutive training sessions. All the exercise sessions were supervised by research assistants. The subjects in the control group did not perform any physical activity throughout the study period.

\section{Data analysis}

The sample size was calculated by $G^{*}$ Power 3.1.0 software, given an alpha level at 0.05 , an effect size of 0.40 , and power at 0.80 . The appropriate sample size was estimated to be 15 subjects for the

Table 1. Physical characteristics of subjects at baseline (PRE) and after 4 weeks of aerobic exercise intervention (POST)

\begin{tabular}{lccccc}
\hline \multirow{2}{*}{ Variable } & \multicolumn{2}{c}{ EX $(\mathrm{n}=16)$} & & \multicolumn{2}{c}{ CON $(\mathrm{n}=19)$} \\
\cline { 2 - 3 } \cline { 5 - 6 } & PRE & POST & & PRE & POST \\
\hline Age $(\mathrm{yr})$ & $39.4 \pm 3.2$ & & & $40.3 \pm 3.7$ & \\
Height $(\mathrm{cm})$ & $162.7 \pm 5.6$ & - & & $161.5 \pm 4.9$ & - \\
Weight $(\mathrm{kg})$ & $89.2 \pm 14.1$ & $89.0 \pm 14.3$ & & $90.4 \pm 15.3$ & $90.3 \pm 15.6$ \\
BMl $\left(\mathrm{kg} / \mathrm{m}^{2}\right)$ & $34.2 \pm 4.6$ & $34.0 \pm 4.9$ & & $34.7 \pm 4.8$ & $34.6 \pm 5.3$ \\
\% Body fat $(\%)$ & $42.1 \pm 5.2$ & $41.9 \pm 5.4$ & & $42.6 \pm 5.2$ & $42.5 \pm 5.8$ \\
VAT $(\mathrm{kg})$ & $0.8 \pm 0.2$ & $0.9 \pm 0.3$ & & $0.9 \pm 0.3$ & $0.9 \pm 0.3$ \\
VO & $23.5 \pm 4.1$ & $26.7 \pm 4.9_{\text {max }}(\mathrm{mL} / \mathrm{kg} / \mathrm{min})$ & & $22.9 \pm 4.1$ & $23.3 \pm 4.8^{\# \#}$ \\
\hline
\end{tabular}

Values are presented as mean \pm standard deviation.

EX, exercise group; CON, control group; BMI, body mass index; VAT, visceral adipose tissue mass; $\mathrm{VO}_{2 \max }$, maximal oxygen consumption.

${ }^{* *}$ Significantly different from PRE within group $(P<0.01)$. ${ }^{\# \text { Significantly different }}$ from EX at the same measurement point $(P<0.01)$. current study. All statistical analyses were conducted using Sigmaplot 13 (Systat Software, Inc., San Jose, CA, USA). Two-way analysis of variance (ANOVA) was used to analyze changes in body composition, inflammatory cytokines, and markers of oxidative stress. post hoc tests were performed using Tukey post hoc test and statistical significance was set at $P<0.05$.

\section{RESULTS}

We conducted two-way repeated measures ANOVA with Tukey post hoc test since all variables in this study passed Shapiro-Wilk normality test and Brown-Forsythe equal variance test. Results of these two tests indicate that there is no baseline difference found in all variables measured in this study. Significant time and interaction effects were found only in $\mathrm{VO}_{2 \max }$, TNF- $\alpha$, and TAS.

The physical characteristics of 35 subjects at baseline (PRE) and after the 4 weeks of exercise intervention (POST) are presented in Table 1. There were no significant differences found between EX and CON groups at PRE. EX group observed an increase in $\mathrm{VO}_{2 \max }$ (time $\times$ interaction: $P<0.001, F=9.265$ ) at POST along with no changes in body weight, BMI, \% body fat, and visceral adipose tissue mass. None of these variables were significantly changed in $\mathrm{CON}$ group over the 4-week intervention.

Changes in inflammatory cytokines and markers of oxidative stress are found in Table 2. EX group observed a significant decrease in TNF- $\alpha$ (time $\times$ interaction: $P=0.033, F=3.742$ ) and an increase in TAS (time $\times$ interaction: $P=0.0298, F=4.12$ ) at POST, meanwhile, levels of adiponectin and CRP were unaltered. CON group did not observe any changes in these variables.

Table 2. Changes in markers of inflammation an oxidative stress at baseline (PRE) and after 4 weeks of aerobic exercise intervention (POST)

\begin{tabular}{lcclcc}
\hline \multirow{2}{*}{ Variables } & \multicolumn{2}{c}{ EX $(\mathrm{n}=16)$} & & \multicolumn{2}{c}{$\operatorname{CON}(\mathrm{n}=19)$} \\
\cline { 2 - 3 } \cline { 5 - 6 } & PRE & POST & & PRE & POST \\
\hline Adiponectin $(\mu \mathrm{g} / \mathrm{mL})$ & $6.21 \pm 0.24$ & $6.30 \pm 0.29$ & & $6.29 \pm 0.22$ & $6.29 \pm 0.25$ \\
CRP $(\mathrm{mg} / \mathrm{L})$ & $4.13 \pm 0.25$ & $4.12 \pm 0.26$ & & $4.07 \pm 0.26$ & $4.08 \pm 0.27$ \\
TNF- $\alpha(\mathrm{pg} / \mathrm{mL})$ & $4.57 \pm 0.24$ & $4.29 \pm 0.28^{*}$ & & $4.49 \pm 0.29$ & $4.55 \pm 0.33^{*}$ \\
TAS $(\mathrm{mM})$ & $1.76 \pm 0.10$ & $1.92 \pm 0.10^{*}$ & & $1.77 \pm 0.10$ & $1.78 \pm 0.10^{*}$ \\
8-OHdG $(\mathrm{ng} / \mathrm{mL})$ & $1.92 \pm 0.21$ & $1.87 \pm 0.26$ & & $1.88 \pm 0.28$ & $1.88 \pm 0.32$ \\
\hline
\end{tabular}

Values are presented as mean \pm standar error.

EX, exercise group; CON, control group; CRP, C-reactive protein; TNF- $\alpha$, tumor necrosis factor-alpha; TAS, total antioxidant status; 8-OHdG, 8-hydroxydeoxyguanosine.

${ }^{*}$ Significantly different from PRE within group $(P<0.05)$. "Significantly different from $\mathrm{CON}$ at the same measurement point $(P<0.05)$. 


\section{DISCUSSION}

The present study investigated effects of a 4-week treadmill exercise training at moderate intensity on changes in \% body fat, visceral adipose tissue mass, and markers of inflammation and oxidative stress independently from fat loss in middle-aged obese females. The 4-week exercise training resulted in a significant decrease in TNF- $\alpha$ and elevation of TAS in EX along with an increase in $\mathrm{VO}_{2 \max }$ when no changes were found in body weight, BMI, \% body fat, and visceral adipose tissue mass.

Obesity is known as a risk factor for cardiovascular diseases and metabolic syndrome, which may contribute to increases in circulating inflammatory markers (Beavers et al., 2015; Bruun et al., 2006; de Ferranti and Mozaffarian, 2008) and oxidative stress (Dennis et al., 2013; Zguira et al., 2019) in blood. Regular exercise training at low to moderate intensity is considered as an effective therapeutical treatment of the deleterious effects of obesity (Alcazar et al., 2019; Simioni et al., 2018).

Beneficial effects of exercise training to oxidative stress have been reported in previous studies, in which changes in markers of oxidative stress and antioxidant status were observed following a 24 weeks of endurance exercise training in adults (Park et al., 2005), a 16-week moderate exercise training in elderly population ( $\mathrm{Fa}$ touros et al., 2004; Mota et al., 2019), a 6-week physical activity in children (Kelishadi et al., 2008), and a 3-week high-intensity interval training in young male (Bogdanis et al., 2013). However, it is not clear whether these beneficial effects of exercise training occur independently from changes in fat mass because the relation between oxidative stress and fat loss was not reported in these studies. For instance, reductions in body weight following exercise training were observed (Fatouros et al., 2004; Kelishadi et al., 2008), body weight information was not provided in other two studies (Bogdanis et al., 2013; Park et al., 2005), and the change in fat mass was not measured (Mota et al., 2019) in the studies above.

Moreover, results of recent studies indicate that a decrease in oxidative stress is associated with reductions in $\%$ body fat and fat mass. Kelly et al. (2007) following an 8 weeks of endurance exercise training in overweight children found no changes in fat mass and a marker of oxidative stress, 8-isoprostane. This result is supported by Kanikowska et al. (2021) who investigated effects of an 8 weeks of caloric restriction on changes in fat mass and markers of oxidative stress and found that significant decreases in oxidative stress markers were accompanied by reductions in fat mass and $\%$ body fat in this study.

We found only two studies reported the therapeutic effect of regular exercise on oxidative stress in the absence of fat loss (Samjoo et al., 2013; Vincent et al., 2006). Samjoo et al. (2013) observed decreases in markers of oxidative stress following 3-month cycling exercise training in obese men in the absence of changes in \% body fat and fat mass, emphasizing therapeutic effects of endurance exercise training by enhancing antioxidant capacity and reducing oxidative stress in blood. A similar result was found following a 6-month resistance exercise training in obese older adults (Vincent et al., 2006), in which markers of oxidative stress decreased following resistance training along with improvement in muscular strength and cardiovascular function.

In the present study, we observed that the level of TAS elevated after 4 weeks of moderate-intensity training along with an increase in $\mathrm{VO}_{2 \max }$ when $\%$ body fat and VAT were not changed. Results of the present study correspond to previous studies (Samjoo et al., 2013; Vincent et al., 2006), indicating that an enhancement in physical fitness may contribute, at least a part, to the increase in TAS regardless of changes in fat mass. Vezzoli et al. (2014) suggest for this result that repeated-aerobic exercise training induces antioxidant enzyme adaptation, therefore, reduces reactive oxygen species, and increases antioxidant defenses.

It is generally accepted that proinflammatory cytokines have a direct correlation with increased level of obesity, which causes an increase in the volume and number of adipocytes produced resulting in the creation of a proinflammatory state in the human body (Beavers et al., 2015; Marcell et al., 2005; Nicklas et al., 2004). In contrast, some studies reported that inflammatory status can be improved with lifestyle interventions such as exercise (Balducci et al., 2010; You et al., 2004), diet (Rajaram et al., 2010) or combination of exercise and diet (Lambert et al., 2008). However, the mechanisms by which each lifestyle intervention positively affects inflammatory cytokines are unclear.

Recent studies reported that the reductions of proinflammatory cytokines are not achieved by the exercise interventions but are directly associated with loss of fat mass (Beavers et al., 2015), suggesting that more than $5 \%$ of fat loss is required to observe significant reductions in proinflammatory cytokines. Two other studies found that an elevation of adiponectin (Marcell et al., 2005) and decreases in proinflammatory cytokines (Nicklas et al., 2004) are not associated with the improvement of physical fitness, but positively related to the weight loss. Results of these studies suggest that exercise training alone does not change the levels of inflammatory cytokines. Furthermore, it is not clear whether changes in inflammatory cytokines following exercise training is caused by physiological benefits of exercise training or by weight loss ac- 
companied to exercise since most of the studies that reported significant changes in inflammatory cytokines by exercise training reported weight loss and/or fat loss concomitantly.

Nonetheless, a few studies recently reported the independent effects of exercise training on inflammatory cytokines from more controlled experiments. You et al. (2004) found that the diet plus exercise intervention decreased CRP and TNF- $\alpha$, while the diet only group failed to show the same result although the similar amount of reduction in fat mass was observed. A similar result was reported by Balducci et al. (2010), which observed that 12 months of exercise programs at high intensity (aerobic or aerobic+resistance exercises) significantly reduced levels of proinflammatory cytokines such as CRP and TNF- $\alpha$ and increased the level of adiponectin even in the absence of changes in body weight and fat mass, while these changes were not observed in low-intensity aerobic exercise or control group. It is suggested that regular exercise can significantly attenuate mortality risk in obese individuals even in the absence of fat loss, especially when the exercise intensity is high.

Current evidence provides potential mechanisms by which exercise trainings, independently from fat loss, may suppress the production of inflammatory markers (Shaw et al., 2018; Starkie et al., 2003), and enhance the anti-inflammatory indices (You et al., 2013). Starkie et al. (2003) observed elevations of interleukin-6 (IL-6) and epinephrine and a decrease in TNF- $\alpha$ during exercise as compared to rest and suggested that an exercise-induced elevation of IL-6 in the exercising muscles be released into the systemic circulation, therefore, may inhibit TNF- $\alpha$ production in the blood. Indirect evidence in animal studies supports that elevation of IL-6 is tightly linked to TNF- $\alpha$ appearance (Fischer, 2006).

Even though epinephrine was not measured in the present study, exercise-induced increase in epinephrine may suppress the expression of proinflammatory cytokines such as TNF- $\alpha$ and CRP by increasing IL- 4 and IL-10, and decreasing IL-12 in the blood circulation (Agarwal and Marshall, 2000; Shaw et al., 2018). It corresponds to van der Poll et al. (1996) who found epinephrine infusions to healthy male subjects increased level of IL-10 and attenuated TNF- $\alpha$ secretion in response to endotoxin administration.

Evidence from animal studies indicates that exercise training may reduce expression of proinflammatory cytokines from adipose tissue independent of its effects of fat mass. It was found in animal studies that exercise training increased the capillary density in visceral adipose tissue (Czarkowska-Paczek et al., 2011; Hatano et al., 2011) and reduced vasoconstriction factors (Pereira et al., 2009), and elevated blood flow in adipose tissue consequently (Enevold- sen et al., 2000). Thereby, it is plausible that exercise training may reduce hypoxia and associated proinflammatory production in adipose tissue in the absence of fat loss, even though there is no human study conducted yet.

In the present study, CRP and adiponectin did not change following the 4 weeks of moderate-intensity exercise training. It is probable that changes in CRP and adiponectin may require a certain duration of exercise training or at least moderate amount of weight loss as shown in other studies (Marcell et al., 2005; Shephard, 2002) and increases in adiponectin (Bouassida et al., 2010; Marcell et al., 2005).

There are two potential limitations to this study that must be considered. Firstly, daily diet intake was not controlled in this study even though subjects were asked to maintain their lifestyle during the 4-week intervention period. Therefore, it is possible that unchanged body weight and \% body fat in exercise group may be caused by an additional caloric intake due to an increased energy expenditure. Secondly, this study was conducted in a predominantly Hispanic area and focused on Hispanic female adults, therefore it cannot be fully concluded whether aerobic exercise trainings can independently contribute to changes in inflammatory cytokines and oxidative stress in other demographics under the condition in which body weight and \% body fat were unaltered.

In conclusions, the present study found that that a positive improvement in inflammatory and oxidative status can be observed following a short term, 4-week aerobic exercise training at moderate intensity in middle-aged obese female before any significant changes in body weight or fat mass were occurred. This result suggests that regular aerobic exercise may be able to contribute, at least a part, to improvement in inflammation and oxidative stress independently from a change in fat mass. These findings provide a clinical evidence that exercise can be used as a potential therapeutic intervention for overweight and obese individuals to prevent the prevalence of chronic diseases. More practically, it is believed that results of the present study may increase adherence rate to regular physical activities in overweight and obese populations even they may not reduce body weight or $\%$ body fat from physical activities. In addition, the present study also confirmed that changes in CRP and adiponectin may need a longer duration of training or may require a significant change in fat mass. Further investigations should be conducted to investigate the potential mechanisms of exercise training associated with inflammatory cytokines and markers of oxidative stress to elucidate the independent role of aerobic exercise training in overweight and obese population. 


\section{CONFLICT OF INTEREST}

No potential conflict of interest relevant to this article was reported.

\section{ACKNOWLEDGMENTS}

This study was supported by Texas A\&M International University Research Grant.

\section{REFERENCES}

Abramson JL, Vaccarino V. Relationship between physical activity and inflammation among apparently healthy middle-aged and older US adults. Arch Intern Med 2002;162:1286-1292.

Adamopoulos S, Parissis J, Kroupis C, Georgiadis M, Karatzas D, Karavolias G, Koniavitou K, Coats AJ, Kremastinos DT. Physical training reduces peripheral markers of inflammation in patients with chronic heart failure. Eur Heart J 2001;22:791-797.

Agarwal SK, Marshall GD Jr. Beta-adrenergic modulation of human type1/type-2 cytokine balance. J Allergy Clin Immunol 2000;105(1 Pt 1): 91-98.

Alcazar J, Losa-Reyna J, Rodriguez-Lopez C, Navarro-Cruz R, Alfaro-Acha A, Ara I, García-García FJ, Alegre LM, Guadalupe-Grau A. Effects of concurrent exercise training on muscle dysfunction and systemic oxidative stress in older people with COPD. Scand J Med Sci Sports 2019; 29:1591-1603.

Balducci S, Zanuso S, Nicolucci A, De Feo P, Cavallo S, Cardelli P, Fallucca S, Alessi E, Fallucca F, Pugliese G, Italian Diabetes Exercise Study (IDES) Investigators. Effect of an intensive exercise intervention strategy on modifiable cardiovascular risk factors in subjects with type 2 diabetes mellitus: a randomized controlled trial: the Italian Diabetes and Exercise Study (IDES). Arch Intern Med 2010;170:1794-1803.

Beavers KM, Beavers DP, Newman JJ, Anderson AM, Loeser RF Jr, Nicklas BJ, Lyles MF, Miller GD, Mihalko SL, Messier SP. Effects of total and regional fat loss on plasma CRP and IL-6 in overweight and obese, older adults with knee osteoarthritis. Osteoarthritis Cartilage 2015;23: 249-256.

Bell JA, Kivimaki M, Hamer M. Metabolically healthy obesity and risk of incident type 2 diabetes: a meta-analysis of prospective cohort studies. Obes Rev 2014;15:504-515.

Bogdanis GC, Stavrinou P, Fatouros IG, Philippou A, Chatzinikolaou A, Draganidis D, Ermidis G, Maridaki M. Short-term high-intensity interval exercise training attenuates oxidative stress responses and improves antioxidant status in healthy humans. Food Chem Toxicol

\section{3;61:171-177.}

Bouassida A, Chamari K, Zaouali M, Feki Y, Zbidi A, Tabka Z. Review on leptin and adiponectin responses and adaptations to acute and chronic exercise. Br J Sports Med 2010;44:620-630.

Bruun JM, Helge JW, Richelsen B, Stallknecht B. Diet and exercise reduce low-grade inflammation and macrophage infiltration in adipose tissue but not in skeletal muscle in severely obese subjects. Am J Physiol Endocrinol Metab 2006;290:E961-E967.

Cao H. Adipocytokines in obesity and metabolic disease. J Endocrinol 2014;220:T47-T59.

Church TS, Barlow CE, Earnest CP, Kampert JB, Priest EL, Blair SN. Associations between cardiorespiratory fitness and C-reactive protein in men. Arterioscler Thromb Vasc Biol 2002;22:1869-1876.

Conraads VM, Beckers P, Bosmans J, De Clerck LS, Stevens WJ, Vrints CJ, Brutsaert DL. Combined endurance/resistance training reduces plasma TNF- $\alpha$ receptor levels in patients with chronic heart failure and coronary artery disease. Eur Heart J 2002;23:1854-1860.

Czarkowska-Paczek B, Zendzian-Piotrowska M, Bartlomiejczyk I, Przybylski J, Gorski J. The influence of physical exercise on the generation of TGF- $\beta 1$, PDGF-AA, and VEGF-A in adipose tissue. Eur J Appl Physiol 2011;111:875-881.

de Ferranti S, Mozaffarian D. The perfect storm: obesity, adipocyte dysfunction, and metabolic consequences. Clin Chem 2008;54:945-955.

Dennis BA, Ergul A, Gower BA, Allison JD, Davis CL. Oxidative stress and cardiovascular risk in overweight children in an exercise intervention program. Child Obes 2013;9:15-21.

Enevoldsen LH, Stallknecht B, Fluckey JD, Galbo H. Effect of exercise training on in vivo lipolysis in intra-abdominal adipose tissue in rats. Am J Physiol Endocrinol Metab 2000;279:E585-E592.

Fatouros IG, Jamurtas AZ, Villiotou VA, Pouliopoulou S, Fotinakis P, Taxildaris K, Deliconstantinos GE. Oxidative stress responses in older men during endurance training and detraining. Med Sci Sports Exerc 2004; 36:2065-2072.

Fischer CP. Interleukin-6 in acute exercise and training: what is the biological relevance? Exerc immunol Rev 2006;12:6-33.

Hatano D, Ogasawara J, Endoh S, Sakurai T, Nomura S, Kizaki T, Ohno $\mathrm{H}$, Komabayashi T, Izawa T. Effect of exercise training on the density of endothelial cells in the white adipose tissue of rats. Scand J Med Sci Sports 2011;21:e115-e121.

Hatoum IJ, Stylopoulos N, Vanhoose AM, Boyd KL, Yin DP, Ellacott KL, Ma LL, Blaszczyk K, Keogh JM, Cone RD, Farooqi IS. Melanocortin-4 receptor signaling is required for weight loss after gastric bypass surgery. J Clin Endocrinol Metab 2012;97:E1023-E1031.

Kanikowska D, Kanikowska A, Swora-Cwynar E, Grzymisławski M, Sato M, Bręborowicz A, Witowski J, Korybalska K. Moderate caloric restric- 
tion partially improved oxidative stress markers in obese humans. Antioxidants (Basel) 2021;10:1018.

Kelishadi R, Hashemi M, Mohammadifard N, Asgary S, Khavarian N. Association of changes in oxidative and proinflammatory states with changes in vascular function after a lifestyle modification trial among obese children. Clin Chem 2008;54:147-153.

Kelly AS, Steinberger J, Olson TP, Dengel DR. In the absence of weight loss, exercise training does not improve adipokines or oxidative stress in overweight children. Metabolism 2007;56:1005-1009.

Lambert CP, Wright NR, Finck BN, Villareal DT. Exercise but not diet-induced weight loss decreases skeletal muscle inflammatory gene expression in frail obese elderly persons. J Appl Physiol 2008;105:473-478.

Marcell TJ, McAuley KA, Traustadóttir T, Reaven PD. Exercise training is not associated with improved levels of $\mathrm{C}$-reactive protein or adiponectin. Metabolism 2005;54:533-541.

Mota MP, Dos Santos ZA, Soares JF, de Fátima Pereira A, João PV, Gaivão IO, Oliveira MM. Intervention with a combined physical exercise training to reduce oxidative stress of women over 40 years of age. Exp Gerontol 2019;123:1-9.

Nicklas BJ, Ambrosius W, Messier SP, Miller GD, Penninx BW, Loeser RF, Palla S, Bleecker E, Pahor M. Diet-induced weight loss, exercise, and chronic inflammation in older, obese adults: a randomized controlled clinical trial. Am J Clin Nutr 2004;79:544-551.

Park JY, Ferrell RE, Park JJ, Hagberg JM, Phares DA, Jones JM, Brown MD. $\mathrm{NADPH}$ oxidase p22phox gene variants are associated with systemic oxidative stress biomarker responses to exercise training. J Appl Physiol 2005;99:1905-1911.

Pereira MG, Ferreira JC, Bueno CR, Mattos KC, Rosa KT, Irigoyen MC, Oliveira EM, Krieger JE, Brum PC. Exercise training reduces cardiac angiotensin II levels and prevents cardiac dysfunction in a genetic model of sympathetic hyperactivity-induced heart failure in mice. Eur J Appl Physiol 2009;105:843-850.

Pérez-Escamilla R, Lutter CK, Rabadan-Diehl C, Rubinstein A, Calvillo A, Corvalán C, Batis C, Jacoby E, Vorkoper S, Kline L, Ewart-Pierce E. Prevention of childhood obesity and food policies in Latin America: from research to practice. Obes Rev 2017;18:28-38.

Rajaram S, Connell KM, Sabaté J. Effect of almond-enriched high-monounsaturated fat diet on selected markers of inflammation: a randomised, controlled, crossover study. Br J Nutr 2010;103:907-912.

Samjoo IA, Safdar A, Hamadeh MJ, Raha S, Tarnopolsky MA. The effect of endurance exercise on both skeletal muscle and systemic oxidative stress in previously sedentary obese men. Nutr Diabetes 2013;3:e88.

Shaw DM, Merien F, Braakhuis A, Dulson D. T-cells and their cytokine production: the anti-inflammatory and immunosuppressive effects of strenuous exercise. Cytokine 2018;104:136-142.

Shephard RJ. Cytokine responses to physical activity, with particular reference to IL-6: sources, actions, and clinical implications. Crit Rev Immunol 2002;22:165-182.

Simioni C, Zauli G, Martelli AM, Vitale M, Sacchetti G, Gonelli A, Neri LM. Oxidative stress: role of physical exercise and antioxidant nutraceuticals in adulthood and aging. Oncotarget 2018;9:17181-17198.

Starkie R, Ostrowski SR, Jauffred S, Febbraio M, Pedersen BK. Exercise and IL- 6 infusion inhibit endotoxin-induced TNF- $\alpha$ production in humans. FASEB J 2003;17:884-886.

Tumova E, Sun W, Jones PH, Vrablik M, Ballantyne CM, Hoogeveen RC. The impact of rapid weight loss on oxidative stress markers and the expression of the metabolic syndrome in obese individuals. J Obes 2013;2013:729515.

van der Poll T, Coyle SM, Barbosa K, Braxton CC, Lowry SF. Epinephrine inhibits tumor necrosis factor-alpha and potentiates interleukin 10 production during human endotoxemia. J Clin Invest 1996;97:713-719.

van Rossum EF. Obesity and cortisol: new perspectives on an old theme. Obesity (Silver Spring) 2017;25:500-501.

Vezzoli A, Pugliese L, Marzorati M, Serpiello FR, La Torre A, Porcelli S. Time-course changes of oxidative stress response to high-intensity discontinuous training versus moderate-intensity continuous training in masters runners. PLoS One 2014;9:e87506.

Vincent HK, Bourguignon C, Vincent KR. Resistance training lowers exercise-induced oxidative stress and homocysteine levels in overweight and obese older adults. Obesity (Silver Spring) 2006;14:1921-1930.

Wang T, He C. Pro-inflammatory cytokines: The link between obesity and osteoarthritis. Cytokine Growth Factor Rev 2018;44:38-50.

You T, Arsenis NC, Disanzo BL, LaMonte MJ. Effects of exercise training on chronic inflammation in obesity. Sports Med 2013;43:243-256.

You T, Berman DM, Ryan AS, Nicklas BJ. Effects of hypocaloric diet and exercise training on inflammation and adipocyte lipolysis in obese postmenopausal women. J Clin Endocrinol Metab 2004;89:1739-1746.

Zatterale F, Longo M, Naderi J, Raciti GA, Desiderio A, Miele C, Beguinot F. Chronic adipose tissue inflammation linking obesity to insulin resistance and type 2 diabetes. Front Physiol 2020;10:1607.

Zguira MS, Slimani M, Bragazzi NL, Khrouf M, Chaieb F, Saïag B, Tabka Z. Effect of an 8-week individualized training program on blood biomarkers, Adipokines and endothelial function in obese Young adolescents with and without metabolic syndrome. Int J Environ Res Public Health 2019;16:751. 